TITLE:

\title{
The presence of heterospecific males causes an Allee effect
}

$\operatorname{AUTHOR}(\mathrm{S})$ :

Kyogoku, Daisuke; Nishida, Takayoshi

CITATION:

Kyogoku, Daisuke ...[et al]. The presence of heterospecific males causes an Allee effect. Population Ecology 2012, 54(3): 391-395

ISSUE DATE:

2012-07

URL:

http://hdl.handle.net/2433/159430

\section{RIGHT:}

The final publication is available at www.springerlink.com; この論文は 出版社版でありません。引用の際には出版社版をご確認ご利用くださ $\omega_{\circ}$; This is not the published version. Please cite only the published version. 
1 Title: The presence of heterospecific males causes an Allee effect.

2

3 Authors: Daisuke Kyogoku* and Takayoshi Nishida

$4 *$ Author for correspondence

5 Laboratory of Insect Ecology, Graduate School of Agriculture, Kyoto University,

$6 \quad$ Kyoto, Japan.

$7 \quad$ E-mail: kyogoku@kais.kyoto-u.ac.jp

8

Address: Kitashirakawa-oiwakecho, Sakyo, Kyoto, Kyoto 606-8502, Japan.

$9 \quad$ Takayoshi Nishida

10 Laboratory of Insect Ecology, Graduate School of Agriculture, Kyoto University,

11 Kyoto, Japan.

Present affiliation: Department of Ecosystem Studies, School of Environmental

13 Science, University of Shiga Prefecture, Hikone, Japan.

$14 \quad$ E-mail: nishida.t@ses.usp.ac.jp

$16 \quad$ Number of text pages; 12

17 Number of figures and tables: One figure and no table. 


\section{Abstract}

21 The Allee effect is a positive causal relationship between any component of fitness and population density or size. Allee effects strongly affect the persistence of small or sparse populations. Predicting Allee effects remains a challenge, possibly because not all causal mechanisms are known. We hypothesized that reproductive interference (an interspecific reproductive interaction that reduces the fitness of the species involved) can generate an Allee effect. If the density of the interfering species is constant, an increase in the population of the species receiving interference may dilute the per capita effect of reproductive interference and may generate an Allee effect. To test this hypothesis, we examined the effect of heterospecific males on the relationship between

30 per capita fecundity and conspecific density in Callosobruchus chinensis and $C$. maculatus. Of the two species, only $C$. maculatus females suffer reproductive interference from heterospecific males. Only C. maculatus, the species susceptible to reproductive interference, demonstrated an Allee effect, and only when heterospecific males were present. In contrast, $C$. chinensis, the species not susceptible to reproductive interference, demonstrated no Allee effect regardless of the presence of heterospecific 
36 males. Our results show that reproductive interference in fact generated an Allee effect,

37 suggesting the potential importance of interspecific sexual interactions especially in small or sparse populations, even in the absence of a shared resource. It may be possible to predict Allee effects produced by this mechanism a priori by testing reproductive interference between closely related species.

\section{Key words}

Callosobruchus $\bullet$ Depensation $・$ Dilution effect $•$ Frequency dependence $・$ Interspecific interaction $・$ Reproductive interference

\section{Introduction}

The Allee effect is a positive causal relationship between any component of fitness and population density or size (Stephens et al. 1999). Allee effects have been broadly

51 recognized as an important ecological phenomenon in conservation biology

52 (Courchamp et al. 1999; Stephens and Sutherland 1999), biological invasion (Taylor and 
and Roush 1993), because they can lead to the extinction of small or sparse populations.

Various mechanisms have been suggested to generate Allee effects, including mate-finding difficulty, lack of predator satiation, and reduced foraging efficiency (Courchamp et al. 2008). However, though some species are unlikely to suffer from an Allee effect, other species with similar life history traits are subject to Allee effects (e.g., Myers et al. 1995; Drake 2005; Fauvergue et al. 2007). Thus, predicting which species will be subject to an Allee effect remains a challenge. It is possibly because some of Allee effects are caused by an as yet unknown mechanism.

We propose the novel hypothesis that reproductive interference can generate an Allee effect, owning to its positive frequency dependence. Reproductive interference is a negative interspecific sexual interaction that reduces the fitness of at least one of the species involved (Gröning and Hochkirch 2008; Burdfield-Steel and Shuker 2011). For example, when a male of species A mistakenly recognizes a female of species B as a conspecific and attempts to mate with her, the female might suffer from an energy or time loss, reduced opportunities to mate with conspecifics, gamete wastage, genital damage, or hybrid formation (Gröning and Hochkirch 2008). Notably, in terms of its effect on population dynamics, reproductive interference seems to show frequency dependence (Kuno 1992; Gröning and Hochkirch 2008; Takakura et al. 2009): the 
72 higher the frequency of species A, the greater the intensity of the reproductive interference on species B. If for a pair of species, reproductive interference is asymmetric (unidirectional) and the density of interfering species A is constant, the per capita level of interference with the interfered species B should become diluted as the density of species B increases. This dilution of reproductive interference should generate an Allee effect when it is accompanied by increased fitness of species B. Thus, reproductive interference may generate an Allee effect via a dilution effect, just as predation does (Gascoigne and Lipcius 2004). fecundity and conspecific density when heterospecific males were present in a pair of bean weevil species, Callosobruchus chinensis (Linnaeus) and C. maculatus (Fabricius). Nishigaki (1963) measured copulation frequency in C. chinensis and reported that males had difficulty in finding mates. In typical laboratory conditions, however, both $C$. chinensis and $C$. maculatus show no positive relationship between population density and lifetime reproductive success in isolation from the other (e.g., Yoshida 1966). Kishi et al. (2009) showed that the fecundity of $C$. maculatus, but not that of $C$. chinensis, is asymmetrically reduced when heterospecific males are present; thus, they show asymmetric reproductive interference. We predict specifically that $C$. maculatus, the 
90 species more susceptible to reproductive interference, will suffer an Allee effect only

91 when heterospecific males are present, and that $C$. chinensis, the non-susceptible species,

92 suffers no Allee effect.

93

\section{$94 \quad$ Materials and methods}

95

96 We used the jC-F strain of C. chinensis (see Harano and Miyatake 2005) and the hQ

97 strain of C. maculatus (see Miyatake and Matsumura 2004), both of which were derived

98 from the stock cultures of Okayama University. We chose these strains, because Kishi et

99 al. (2009) used these same strains to confirm unilateral reproductive interference by $C$.

100 chinensis on $C$. maculatus. The stock cultures were maintained under laboratory

101 conditions $\left(25^{\circ} \mathrm{C}, 50-60 \%\right.$ relative humidity, and $\left.16 \mathrm{~L} 8 \mathrm{D}\right)$ and fed on adzuki beans,

102 Vigna angularis (Willd.) cv. Dainagon. Mated females of C. chinensis and C. maculatus

103 lay about 60 eggs on bean surfaces without feeding during their adult lifespan of around

10410 days. Males of both species try to copulate with either heterospecific or conspecific

105 females indiscriminately even when conspecific females are present (Kishi et al. 2009).

106 Interspecific copulation is sometimes observed, but no hybrid has been reported

107 (Yamane and Miyatake 2010). 
110 plastic Petri dishes $(\phi 90 \mathrm{~mm} \times 20 \mathrm{~mm})$. Then, about 10 females of each species were

111 randomly chosen from the stock cultures (200-400 individuals in each generation), and

112 allowed to lay eggs on the beans in one of the dishes. Beans with one, two, or three eggs

113 on them were selected and placed individually in wells of a 24 -well plastic plate (each

114 well, $\phi 15.5 \mathrm{~mm} \times 17.3 \mathrm{~mm})$. Beans with four or more eggs were discarded to avoid

115 potential overcrowding effects. Each well was checked every day for newly emerged

116 virgin individuals (i.e., individuals that emerged singly or in a unisexual group).

118 of hatched eggs per female) of $C$. maculatus was investigated under different $C$.

119 maculatus density regimes as follows. From one to five $C$. maculatus pairs, each

120 consisting of one male and one female individual, were housed with three $C$. chinensis males throughout their lives in a plastic Petri dish $(\phi 70 \mathrm{~mm} \times 15 \mathrm{~mm})$ containing $9.0 \mathrm{~g}$

122 of adzuki beans. Before the two species were put together, we allowed each $C$. maculatus female to copulate with a conspecific male to exclude the possibility that the presence of heterospecific males would lead to a potential mate-finding difficulty. We 
126 wished to investigate how reproductive interference by heterospecific males affected the

127 fecundity of the C. maculatus females. As the control, we also examined the per capita

128 fecundity of one to five pairs of $C$. maculatus housed without any $C$. chinensis males.

129 After 20 days, by which time all females had died, we counted the number of hatched

130 eggs in each experimental replication and control treatment (we performed 16 or 17

131 replications of each) in each density regime to determine the per capita fecundity of $C$.

132 maculatus and the effect of reproductive interference by $C$. chinensis males. We

133 similarly examined the effect of $C$. maculatus males on the relationship between

134 fecundity and density in C. chinensis. All experiments were conducted at $25^{\circ} \mathrm{C}, 50-60 \%$

135 relative humidity, and 16L8D. We used analysis of covariance (ANCOVA) to examine

136 the effects of the presence of heterospecific males, conspecific density, and their

137 interactions on per capita fecundity. All statistical analyses were conducted with $\mathrm{R}$

138 software version 2.11.1 (R Development Core Team 2010). The significance level for

139 the statistical tests was set at $P=0.05$.

Results

143 The presence of $C$. chinensis males significantly reduced the per capita fecundity of $C$. 
144 maculatus, and the impact was greater when the density of conspecifics was lower

145 (ANCOVA: $C$. chinensis males, $F_{1,165}=130.05, P<0.001$; density, $F_{1,165}=18.71, P<$

1460.001 ; interaction, $F_{1,165}=16.59, P<0.001$; Fig. 1a). In contrast, in the absence of $C$.

147 chinensis males, density and fecundity of $C$. maculatus were not significantly related

148 (linear regression analysis: $F_{1,83}=0.028, P=0.87$; Fig. 1a). The presence of $C$.

149 maculatus males had no significant effect on per capita fecundity of $C$. chinensis, but $C$.

150 chinensis density had a significant negative effect on the fecundity of that species

151 (ANCOVA: $C$. maculatus males, $F_{1,164}=0.56, P=0.45$; density, $F_{1,164}=8.81, P=$ $152 \quad 0.003$; interaction, $F_{1,164}=2.79, P=0.10$; Fig. 1b).

Discussion

We suggest that reproductive interference by $C$. chinensis generated an Allee effect in $C$.

maculatus. The presence of $C$. chinensis males reduced the per capita fecundity of $C$.

documented asymmetric reproductive interference by $C$. chinensis males on $C$.

maculatus females (Kishi et al. 2009). A higher density of C. maculatus, however, 
162 Although the presence of $C$. chinensis males reduced the per capita fecundity of $C$.

163 maculatus, in the absence of $C$. chinensis, the fecundity of $C$. maculatus did not depend

164 on density (Fig. 1a). Thus, we can attribute the observed fecundity reduction in $C$.

165 maculatus exclusively to the presence of $C$. chinensis males. Callosobruchus chinensis

166 males attempt to mate with females of either species indiscriminately (Kishi et al. 2009).

167 In this study, the relative abundance of $C$. chinensis males to $C$. maculatus decreased as

168 C. maculatus density increased. A single C. maculatus female was, therefore, expected

169 to experience fewer mating attempts by $C$. chinensis males as the density of $C$.

170 maculatus increased, as a result of the dilution effect. If this held true, the hypothesized

171 reduction in the number of mating attempts by $C$. chinensis males can explain the

172 enhanced per capita fecundity of C. maculatus with increasing C. maculatus density

173 (Fig. 1a). We also observed no Allee effect in C. chinensis, which shows little

174 susceptibility to reproductive interference by $C$. maculatus, a result consistent with our

175 hypothesis (Fig. 1b).

asymmetric; although $C$. chinensis males reduced the fecundity of $C$. maculatus (Fig.1a),

C. maculatus males had no significant effect on the fecundity of C. chinensis (Fig. 1b). 
180 females, but it seldom occurs between $C$. maculatus males and $C$. chinensis females

181 (Yamane and Miyatake 2010). Thus, it is likely that the interspecific copulation between

182 C. chinensis males and C. maculatus females plays an important role in the asymmetric

183 reduction in fecundity. Yamane and Miyatake (2010), however, suggested that neither

sperm nor seminal fluid was transferred during interspecific copulation between $C$.

chinensis males and C. maculatus females. In Callosobruchus, male genitalia have

spines, which injure the genital tracts of the females during intraspecific copulation (e.g.,

187 Crudgington and Siva-Jothy 2000; Rönn et al. 2007). The genital spines of $C$. chinensis

males may thus injure the genital tracts of $C$. maculatus females, and the putative injury

might cause the fecundity reduction in $C$. maculatus. Another possibility is interspecific

sexual harassment. Though males of both species attempt to mate with heterospecific

191 females (Kishi et al. 2009), some behavioral differences between these species may

whether structural damage or behavioral interference is actually responsible for the 
198 conspecific sperm (Fig. 1a). However, reproductive interference may cause lack of

199 conspecific sperm. For example, heterospecific matting attempts may interfere with

200 conspecific copulations (Liu et al. 2007). Thus, without copulation with a conspecific

201 male prior to interaction with heterospecifics, the Allee effect might be enhanced. The

202 interaction between reproductive interference and conventional mechanisms of Allee

203 effects is an important topic for further research.

It is not clear to what extent reproductive interference causes Allee effects in

nature. The importance of reproductive interference as a mechanism generating an Allee

effect depends on the generality of reproductive interference itself. The role of

207 reproductive interference in population dynamics, however, has not been appreciated

209 likely when a biological invasion occurs (Burdfield-Steel and Shuker 2011), that is,

210 when originally allopatric species begin to interact with each other. Biological invasions,

211 therefore, may be one of the most promising settings in nature for finding an Allee

212 effect generated by reproductive interference. On the other hand, several studies have

213 documented reproductive interference between originally sympatric species (e.g.,

214 McLain and Pratt 1999; Hettyey and Pearman 2003; Van Gossum et al. 2007), 
Allee effects are increasingly appreciated for their importance in applied

217 ecology problems, such as conservation and biological invasion (Taylor and Hastings

218 2005; Courchamp et al. 2008; Tobin et al. 2011). Here we showed that reproductive

219 interference can indeed generate an Allee effect. If reproductive interference can

220 generate an Allee effect in nature, then programs dealing with small or sparse

221 populations need to examine interactions between closely related species, even those

that do not share a limited resource.

223

224 Acknowledgements

225

226 We are grateful to T. Miyatake for providing the bean weevils. We thank N. Osawa,

227 S. Noriyuki and S. Seiter for useful comments on our manuscript, and S. Oku for

228 statistical advice. This study was partly supported by a Grant-in-Aid for Scientific

229 Research (Ministry of Education, Culture, Sports, Science and Technology of Japan;

230 No. 60208189) to TN.

\section{References}


234 Burdfield-Steel ER, Shuker DM (2011) Reproductive interference. Curr Biol

235 21:R450-R451

236

237 Courchamp F, Clutton-Brock T, Grenfell B (1999) Inverse density dependence and the Allee effect. Trends Ecol Evol 14:405-410

Courchamp F, Berec L, Gascoigne J (2008) Allee effects in ecology and conservation. Oxford University Press, Oxford

Crudgington HS, Siva-Jothy MT (2000) Genital damage, kicking and early death - the battle of the sexes takes a sinister turn in the bean weevil. Nature 407:855-856

Drake J (2005) Risk analysis for species introductions: Forecasting population growth of Eurasian ruffe (Gymnocephalus cernuus). Can J Fish Aquat Sci 
253 Gascoigne JC, Lipcius RN (2004) Allee effects driven by predation. J Appl Ecol

Gröning J, Hochkirch A (2008) Reproductive interference between animal species. Q

Harano T, Miyatake T (2005) Heritable variation in polyandry in Callosobruchus chinensis. Anim Behav 70:299-304

Hettyey A, Pearman PB (2003) Social environment and reproductive interference affect reproductive success in the frog Rana latastei. Behav Ecol 14:294-300

Hopper KR, Roush RT (1993) Mate finding, dispersal, number released, and the success of biological-control introductions. Ecol Entomol 18:321-331 and exclusion in species interactions. J Anim Ecol 78:1043-1049 
271 Kuno E (1992) Competitive exclusion through reproductive interference. Res Popul

Ecol 34:275-284

273

Liebhold AM, Tobin PC (2008) Population ecology of insect invasions and their

Liu S, De Barro PJ, Xu J, Luan J, Zang L, Ruan Y, Wan F (2007) Asymmetric mating interactions drive widespread invasion and displacement in a whitefly. Science 318:1769-1772

280 harassment on the fecundity of a host-specific, seed-eating insect (Neacoryphus bicrucis). Behav Ecol Sociobiol 46:164-170

Miyatake T, Matsumura F (2004) Intra-specific variation in female remating in 
288 Myers RA, Barrowman NJ, Hutchings JA, Rosenberg AA (1995) Population-dynamics

Nishigaki J (1963) The effect of low population density on the mating chance and the

R Development Core Team (2010) R: a language and environment for statistical computing. R Foundation for Statistical Computing, Vienna, Austria. URL: http://www.R-project.org

298

Rönn J, Katvala M, Arnqvist G (2007) Coevolution between harmful male genitalia and female resistance in seed beetles. Proc Natl Acad Sci USA 104:10921-10925 ecology and conservation. Trends Ecol Evol 14:401-405 
Takakura K, Nishida T, Matsumoto T, Nishida S (2009) Alien dandelion reduces the effects. Biol Invasions 11:973-981

Taylor CM, Hastings A (2005) Allee effects in biological invasions. Ecol Lett 8:895-908 biological invasions. Ecol Lett 14:615-624

Tobin PC, Berec L, Liebhold AM (2011) Exploiting Allee effects for managing extracts in two Callosobruchus species: Consequences for interspecific mating. 
324 Yoshida, T (1966) Studies on the interspecific competition between bean weevils.

325 Memoirs of the Faculty of Liberal Arts and Education, Miyazaki University,

326

Miyazaki 20:59-98

327

328 


\section{Figure legend}

331

332 Fig. 1. Per capita fecundity of C. maculatus (a) and C. chinensis (b) in the absence (open circles) or presence (closed circles) of heterospecific males, plotted against population density. In (a), the broken line and the solid line are regression lines for the data in the absence or presence of $C$. chinensis males, respectively. In (b), the regression was calculated using all data, because the effect of $C$. maculatus males on $C$. chinensis fecundity was not significant. 

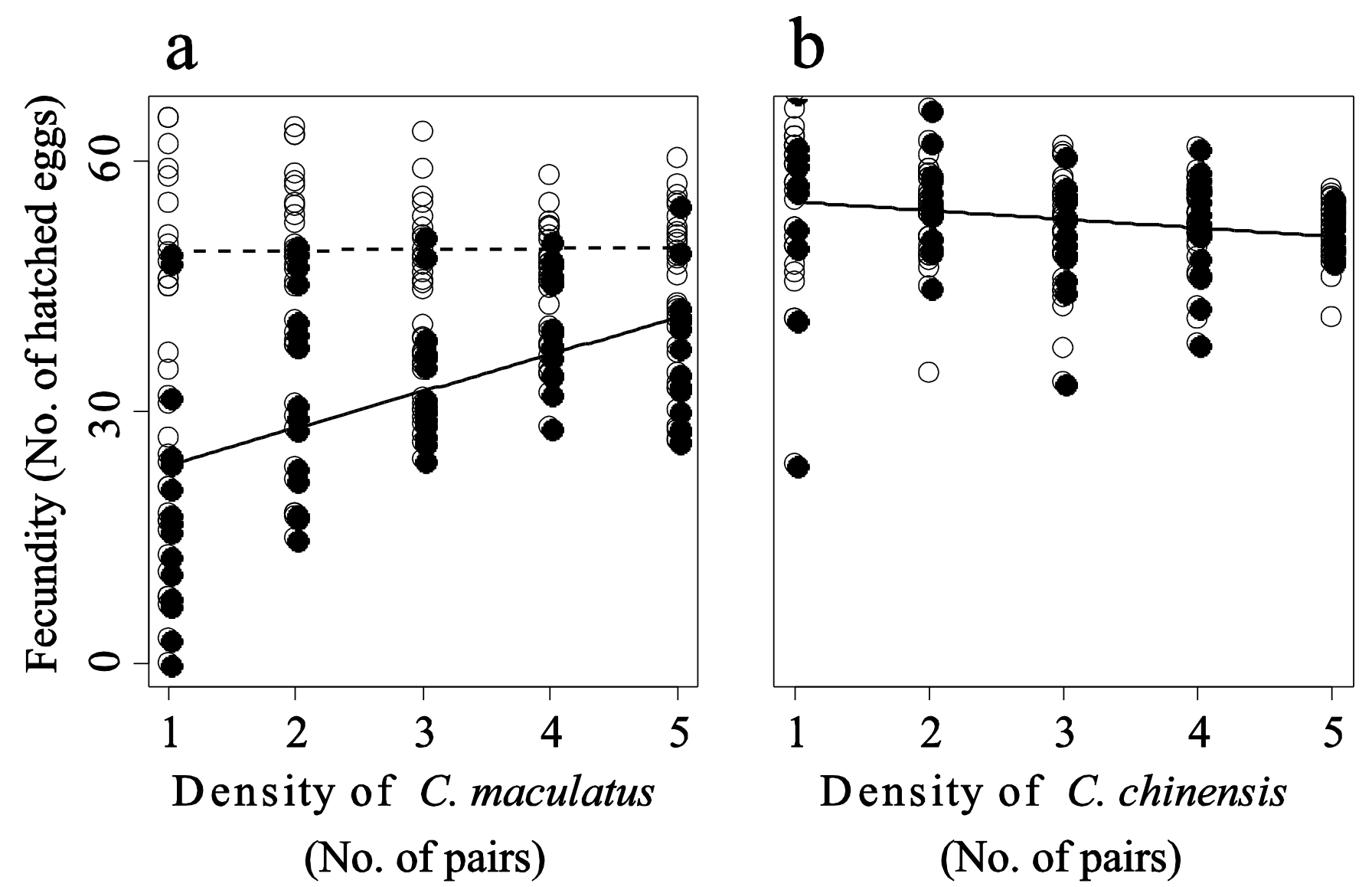